\title{
Soliton propagation in multimode optical fibers
}

\author{
Bruno Crosignani and Paolo Di Porto \\ Fondazione Ugo Bordoni, Istituto Superiore P.T., Viale Europa, Rome, Italy
}

Received November 25, 1980; revised manuscript received March 19, 1981

\begin{abstract}
Soliton propagation in a multimode optical fiber in the presence of an intensity-dependent refractive index is investigated by means of a set of nonlinear coupled equations derived in the frame of coupled-mode theory. In particular, the conditions on modal amplitudes and modal dispersion necessary for soliton existence are derived.
\end{abstract}

The possibility of propagating distortion-free pulses (envelope solitons) in optical waveguides by taking advantage of the quadratic nonlinearity of the refractive index in order to balance material dispersion was pointed out several years ago. ${ }^{1}$ Subsequently, this problem has been the object of a renewed interest that has led to a better understanding of the role of the waveguide structure ${ }^{2}$ and of the finite coherence time of the carrier. ${ }^{3}$

Following the above investigations and after the recent experimental observation of solitons $\mathrm{s}^{4}$ concerning single-mode optical fibers, it is natural to look for a generalization of the previous results to the case in which more than one mode is present. Along this line, the possibility of exploiting nonlinear-mode interaction in a multimode fiber for optical pulse confinement and the conditions under which it can take place have been stated. ${ }^{5}$ We write here, relying on the coupled-modetheory approach to nonlinear propagation, ${ }^{6}$ a general set of coupled equations describing pulse evolution in a multimode optical fiber in the presence of an intensity-dependent refractive index. We specialize this system of equations to investigate soliton propagation, thus enabling us to derive the conditions under which it can be achieved.

In the frame of the coupled-mode formalism, the evolution of a multimode optical pulse is studied by identifying the departure of the refractive index from ideality, which is responsible for coupling, with the presence of a nonlinear part in the refractive index itself. Assuming that the fiber material is isotropic and that the fast-responding electronic processes dominate the nonlinear response, the nonlinear polarization $P^{\mathrm{NL}}$ takes the form ${ }^{7}$

$$
\mathbf{P}^{\mathrm{NL}}(t)=(\sigma / 2) \mathbf{E}(t) \cdot \mathbf{E}(t) \mathbf{E}(t),
$$

which implies a nonlinear contribution to the dielectric constant of the kind

$$
\epsilon_{2}\left|\hat{E}_{x}(t)\right|^{2},
$$

where $\epsilon_{2}=(5 / 8) \sigma$ and $\hat{E}_{x}$ is the analytic representation of the component of the electric field along an arbitrary axis orthogonal to the $z$ direction of propagation. The validity of Eq. (2) relies on the assumption of a circularly symmetric fiber in which random power exchange between the two degenerate polarization states of an ar- bitrary mode takes place over few centimeters and on the hypothesis of weakly guiding fiber allowing one to neglect the longitudinal field component with respect to the transverse ones.

The analytic field $\hat{E}_{x}$ can be written as

$$
\hat{E}_{x}(\mathbf{r}, z, t)=\sum_{\nu} E_{\nu}(\mathbf{r}) \exp \left[i \omega_{\nu} t-i \beta_{\nu}\left(\omega_{\nu}\right) z\right] \hat{\Phi}_{\nu}(z, t),
$$

where $\mathbf{r}=(x, y)$ represents the transverse coordinates, the $E_{\nu}$ 's are the modal transverse configurations, $\beta_{\nu}$ is the propagation constant of the $\nu$ th mode, and the $\hat{\Phi}_{p}(z, t)$ 's are the slowly varying mode amplitudes, having allowed for the various modes to be centered on slightly different frequencies $\omega_{\nu}$. Equation (2) implies a total dielectric constant of the form

$$
\epsilon(\mathbf{r}, z, \omega)=\epsilon_{1}(\mathbf{r}, \omega)+\epsilon_{2}\left|\hat{E}_{x}(\mathbf{r}, z, t)\right|^{2},
$$

where $\epsilon_{1}(\mathbf{r}, \omega)$ is the fiber linear dielectric constant, so that $\epsilon_{2}\left|\hat{E}_{x}\right|^{2}$ can be regarded as the fiber deviation from ideality giving rise to mode coupling. By taking advantage of the results of coupled-mode theory, ${ }^{6}$ one is able to obtain the following set of coupled equations:

$$
\begin{array}{r}
L_{\nu} \hat{\Phi}_{\nu}(z, t)=-2 i\left[\sum_{\mu \neq \nu} R_{\mu \nu}\left|\hat{\Phi}_{\mu}\right|^{2}+(1 / 2) R_{\nu \nu}\left|\hat{\Phi}_{\nu}\right|^{2}\right] \hat{\Phi}_{\nu} \\
\nu=1,2, \ldots,
\end{array}
$$

where

$$
\begin{aligned}
R_{\mu \nu} & =\left[\omega_{0}\left(\epsilon_{2} / \epsilon_{0}\right) / 2 n_{1} c\right] \frac{\iint_{-\infty}^{+\infty} E_{\mu}^{2}(\mathbf{r}) E_{\nu}^{2}(\mathbf{r}) \mathrm{d} x \mathrm{~d} y}{\iint_{-\infty}^{+\infty} E_{\nu}{ }^{2}(\mathbf{r}) \mathrm{d} x \mathrm{~d} y} \\
& \equiv\left[\omega_{0}\left(\epsilon_{2} / \epsilon_{0}\right) / 2 n_{1} c\right] \alpha_{\mu \nu}
\end{aligned}
$$

and

$$
L_{\nu}=\left[\partial / \partial z+\left(1 / V_{\nu}\right) \partial / \partial t-\left(i / 2 A_{\nu}\right) \partial^{2} / \partial t^{2}\right],
$$

with

$$
V_{\nu}^{-1}=\left(\mathrm{d} \beta_{\nu} / \mathrm{d} \omega\right)_{\omega=\omega_{\nu}}, \quad A_{\nu}{ }^{-1}=\left(\mathrm{d}^{2} \beta_{\nu} / \mathrm{d} \omega^{2}\right)_{\omega=\omega_{\nu}},
$$

$\omega_{0}$ being the average frequency of the field and $n_{1}$ the average value of the fiber linear refractive index.

In principle, whenever the $\omega_{\nu}$ 's are chosen in such a 
way as to provide a common group velocity $V$ for the various modes, that is, when

$$
V^{-1}=\left(\mathrm{d} \beta_{1} / \mathrm{d} \omega\right)_{\omega=\omega_{1}}=\left(\mathrm{d} \beta_{2} / \mathrm{d} \omega\right)_{\omega=\omega_{2}}=\ldots,
$$

Eqs. (5) admit of bright-soliton solutions of the kind

$$
\begin{array}{r}
\hat{\Phi}_{\nu}(z, t)=\hat{\Phi}_{0 \nu} \exp \left(i z / 2 A_{\nu} \tau^{2}\right) \operatorname{sech}[(t-z / V) / \tau], \\
\nu=1,2, \ldots,
\end{array}
$$

provided that the amplitudes $\hat{\Phi}_{0 \nu}$ and the duration $\tau$ satisfy the relations

$$
\begin{aligned}
-1 / A_{\nu} \tau^{2}=2 \sum_{\mu} R_{\mu \nu}\left|\hat{\Phi}_{0 \mu}\right|^{2}-R_{\nu \nu}\left|\hat{\Phi}_{0 \nu}\right|^{2}, & \\
\nu & =1,2, \ldots .
\end{aligned}
$$

Equations (11), which imply $A_{\nu}<0$, generalize the usual soliton-existence condition valid for single-mode fibers $^{2,6}$ and are valid for any refractive-index profile $n_{1}(\mathbf{r})$. In particular, recalling that $A_{\nu}$ is nearly independent of $\nu$, a multimode step-index fiber admits of the solution $\left|\hat{\Phi}_{01}\right|^{2}=\left|\hat{\Phi}_{02}\right|^{2}=\ldots=\left|\hat{\Phi}_{0}\right|^{2}$, with

$$
2 R\left|\hat{\Phi}_{0}\right|^{2}=-1 / A \tau^{2}
$$

where $1 / A=\left(\mathrm{d}^{2} k / \mathrm{d} \omega^{2}\right)_{\omega=\omega_{0}}$ indicates the core material dispersion and

$$
R=\left[\omega_{0}\left(\epsilon_{2} / \epsilon_{0}\right) / 2 n_{1} c\right] \sum_{\mu} E_{\mu}^{2}(r=0) .
$$

Equations (12) and (13) were obtained by neglecting the negative contribution on the right-hand side of Eq. (11) and by taking advantage of the relation ${ }^{8}$

$$
\frac{\sum_{\mu} E_{\mu}{ }^{2}(r)}{\sum_{\mu} E_{\mu}{ }^{2}(0)}=\frac{n_{1}^{2}(r)-n_{1}^{2}(a)}{n_{1}^{2}(0)-n_{1}^{2}(a)},
$$

where $a$ is the fiber core radius, specialized to a stepindex fiber. In this case, if the transverse-mode patterns are normalized to unity, one has ${ }^{8}$

$$
\sum_{\mu} E_{\mu}^{2}(0)=\left(\omega_{0}^{2} / 4 \pi c^{2}\right)\left(n_{1}^{2}-n_{2}^{2}\right),
$$

$n_{1}$ and $n_{2}$ indicating, respectively, the core and cladding refractive index.

In most practical situations, all the $\omega_{\nu}$ 's coincide with $\omega_{0}$, so exact soliton solutions do not exist. However, it is possible to obtain approximate solutions by applying the self-confinement condition introduced for multimode fibers. ${ }^{5}$ More precisely, we use the criterion leading to Eq. (7) of Ref. 5, which yields a lower limit for the electromagnetic intensity required for mode trapping. In our case it reads, for a generic refractiveindex profile,

$$
\begin{aligned}
\left(V_{\nu}-V\right)^{2} / V^{2} \ll & -\left(V^{2} / A_{\nu}\right)\left[\omega_{0}\left(\epsilon_{2} / \epsilon_{0}\right) / n_{1} c\right] \\
& \times \sum_{\mu} \alpha_{\mu \nu}\left|\hat{\Phi}_{\mu}\right|^{2}, \quad \nu=1,2, \ldots,
\end{aligned}
$$

where $V$ is the average velocity of the unperturbed modes. The strong inequality ensures that the overlapping among the different modes is always maintained, so it is possible to substitute $1 / V$ for $1 / V_{\nu}$ in the operator $L_{\nu}$ defined by Eq. (7). This in turn imples that Eqs. (5) admit of the soliton solution of the kind given in Eq. (10). Intuitively, Eq. (16) implies that soliton propagation is more easily achieved in graded-index optical fibers whose refractive-index profiles provide small modal dispersion.

Finally, it is worth mentioning the recent development of polarization-maintaining birefringent multimode fibers. ${ }^{9}$ In this case, the results obtained in this Letter still apply, provided that $(3 / 5) \epsilon_{2}$ is substituted for $\epsilon_{2}$.

\section{References}

1. A. Hasegawa and F. Tappert, "Transmission of stationary nonlinear optical pulses in dispersive dielectric fibers. I. Anomalous dispersion," Appl. Phys. Lett. 23, 142-144 (1973).

2. See, for example, B. Bendow, P. D. Gianino, N. Tzoar, and M. Jain, "Theory of nonlinear pulse propagation in optical waveguides," J. Opt. Soc. Am. 70, 539-546 (1980).

3. B. Crosignani, P. Di Porto, and C. H. Papas, "The role of intensity fluctuations in nonlinear pulse propagation," Opt. Lett. 5, 467-468 (1980).

4. L. F. Mollenauer, R. H. Stolen, and J.P. Gordon, "Experimental observation of picosecond pulse narrowing and solitons in optical fibers," Phys. Rev. Lett. 45, 1095-1098 (1980).

5. A. Hasegawa, "Self-confinement of multimode optical pulse in a glass fiber," Opt. Lett. 5, 416-417 (1980).

6. B. Crosignani; P. Di Porto, and C. H. Papas, "Coupledmode theory approach to nonlinear pulse propagation in optical fibers," Opt. Lett. 6, 61-63 (1981).

7. A. Owyoung, "The origins of the nonlinear refractive indices of liquids and glasses," Ph.D. Thesis (California Institute of Technology, Pasadena, Calif., 1971) (unpublished).

8. B. Crosignani, P. Di Porto, and S. Solimeno, "Influence of guiding structures on spontaneous and stimulated emission. Raman scattering in optical fibers," Phys. Rev. A 21, 594-598 (1980).

9. R. H. Stolen, R. E. Wagner, and W. Pleibel, "Linear polarization in birefringent multimode fibers," in Proceedings of the Third International Conference on Integrated Optics and Optical Fiber Communication (Optical Society of America, Washington, D.C., 1981). 